\title{
Front Matter: Volume 6989
}

, "Front Matter: Volume 6989," Proc. SPIE 6989, Photonic Crystal Materials and Devices VIII, 698901 (14 May 2008); doi: 10.1117/12.801847

SPIE. Event: SPIE Photonics Europe, 2008, Strasbourg, France 


\title{
PROCEEDINGS OF SPIE
}

\section{Photonic Crystal Materials and Devices VIII}

\author{
Richard M. De La Rue \\ Ceferino López \\ Michele Midrio \\ Pierre Viktorovitch \\ Editors
}

\section{8-10 April 2008 \\ Strasbourg, France}

Sponsored by

SPIE Europe

Cosponsored by

Alsace International (France)

Conseil Général du Bas-Rhin (France)

Région Alsace (France)

Communauté Urbaine de Strasbourg (France)

\section{Cooperating Organizations}

AFOP-Association Française des Industries de l'Optique et de la Photonique (France) • EOS-European Optical Society (Germany) • EPIC-European Photonics Industry Consortium (France) • ePIXnet (Belgium) IOP_Institute of Physics (United Kingdom) - NEMO—Network of Excellence on Micro-Optics (Belgium) OLAS (Italy) • The OLLA Project (Germany) • OPERA 2015 (Belgium) • PhOREMOST (Ireland) • Photonics Knowledge Transfer Network • (United Kingdom) • Photonics Cluster (United Kingdom) • Photonics4Life (Germany) • Photonics 21 (Germany) RhenaPhotonics Alsace (France)

Published by

SPIE

Volume 6989 
The papers included in this volume were part of the technical conference cited on the cover and title page. Papers were selected and subject to review by the editors and conference program committee. Some conference presentations may not be available for publication. The papers published in these proceedings reflect the work and thoughts of the authors and are published herein as submitted. The publisher is not responsible for the validity of the information or for any outcomes resulting from reliance thereon.

Please use the following format to cite material from this book:

Author(s), "Title of Paper," in Photonic Crystal Materials and Devices VIII, edited by Richard M. De La Rue, Ceferino López, Michele Midrio, Pierre Viktorovitch, Proceedings of SPIE Vol. 6989 (SPIE, Bellingham, WA, 2008) Article CID Number.

ISSN 0277-786X

ISBN 9780819471871

Published by

SPIE

P.O. Box 10, Bellingham, Washington 98227-0010 USA

Telephone +1 3606763290 (Pacific Time) · Fax +1 3606471445

SPIE.org

Copyright (C) 2008, Society of Photo-Optical Instrumentation Engineers

Copying of material in this book for internal or personal use, or for the internal or personal use of specific clients, beyond the fair use provisions granted by the U.S. Copyright Law is authorized by SPIE subject to payment of copying fees. The Transactional Reporting Service base fee for this volume is $\$ 18.00$ per article (or portion thereof), which should be paid directly to the Copyright Clearance Center (CCC), 222 Rosewood Drive, Danvers, MA 01923. Payment may also be made electronically through CCC Online at copyright.com. Other copying for republication, resale, advertising or promotion, or any form of systematic or multiple reproduction of any material in this book is prohibited except with permission in writing from the publisher. The CCC fee code is 0277-786X/08/\$18.00.

Printed in the United States of America.

Publication of record for individual papers is online in the SPIE Digital Library.

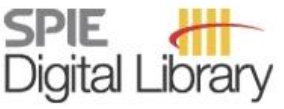

SPIEDigitalLibrary.org

Paper Numbering: Proceedings of SPIE follow an e-First publication model, with papers published first online and then in print and on CD-ROM. Papers are published as they are submitted and meet publication criteria. A unique, consistent, permanent citation identifier (CID) number is assigned to each article at the time of the first publication. Utilization of CIDs allows articles to be fully citable as soon they are published online, and connects the same identifier to all online, print, and electronic versions of the publication. SPIE uses a six-digit CID article numbering system in which:

- The first four digits correspond to the SPIE volume number.

- The last two digits indicate publication order within the volume using a Base 36 numbering system employing both numerals and letters. These two-number sets start with 00, 01, 02, 03, 04 , 05, 06, 07, 08, 09, OA, OB ... 0Z, followed by 10-1Z, 20-2Z, etc.

The CID number appears on each page of the manuscript. The complete citation is used on the first page, and an abbreviated version on subsequent pages. Numbers in the index correspond to the last two digits of the six-digit CID number. 


\section{Contents}

ix Conference Committee

\section{SESSION 1 3D PHOTONIC CRYSTALS AND VOLUME 2D PHOTONIC CRYSTALS}

698903 Light transmission and scattering in engineered colloidal hetero-crystals [6989-02]

B. Ding, M. Bardosova, I. Povey, M. E. Pemble Tyndall National Institute, Univ. College Cork (Ireland); S. G. Romanov, Tyndall National Institute, Univ. College Cork (Ireland) and Ioffe Physical Technical Institute (Russia)

698904 Novel method for fabrication of volume 2D photonic crystals [6989-03]

R. Buczynski, Univ. of Warsaw (Poland); I. Kujawa, Institute of Electronic Materials Technology (Poland); A. Filipkowski, Univ. of Warsaw (Poland); D. Pysz, Institute of Electronic Materials Technology (Poland); F. Hudelist, A. Waddie, Heriot-Watt Univ. (United Kingdom); R. Stepien, Univ. of Warsaw (Poland); M. Taghizadeh, Heriot-Watt Univ. (United Kingdom)

698905 Study of local dispersion in photonic crystal waveguide interfaces and hetero-structures [6989-04]

B. Dastmalchi, Johannes Kepler Univ. Linz (Austria); R. Kheradmand, M. R. A. Monazam, Tabriz Univ. (Iran); A. Hamidipour, Sahand Univ. of Technology (Iran); A. Mohtashami, K. Hingerl, Johannes Kepler Univ. Linz (Austria); J. Zarbakhsh, Johannes Kepler Univ. Linz (Austria) and Kompetenzzentrum Automobil- und Industrie-Elektronik GmbH (Austria)

698906 Two-dimensional and 3D multi-component photonic crystals: theory and experiment [6989-05]

M. F. Limonov, A. V. Baryshev, loffe Physico-Technical Institute (Russia) and Toyohashi Univ. of Technology (Japan); A. B. Khanikaev, M. Inove, Toyohashi Univ. of Technology (Japan); M. V. Rybin, A. K. Samusev, K. B. Samusev, loffe Physico-Technical Institute (Russia); A. V. Sel'kin, loffe Physico-Technical Institute (Russia) and Toyohashi Univ. of Technology (Japan); G. Yushin, Georgia Institute of Technology (USA)

\section{SESSION 2 QUASI PHOTONIC CRYSTALS}

698909 Gap solitons in nonlinear spatiotemporal photonic crystals and gratings [6989-08] F. Biancalana, Cardiff Univ. (United Kingdom); A. Amann, E. P. O'Reilly, Tyndall National Institute (Ireland)

6989 OA Periodically oscillating Anderson localization in random photonic superlattices with resonant units [6989-09]

M. Ghulinyan, Fondazione Bruno Kessler (Italy)

6989 OB Light transport in planar dielectric optical waveguides based on the aperiodic Thue-Morse sequence [6989-10]

M. Hiltunen, VTT Technical Research Ctr. of Finland (Finland); J. Michel, Massachusetts Institute of Technology (USA); L. Dal Negro, Boston Univ. (USA) 
6989 OD Band gap characterization and slow light effects in periodic and quasiperiodic one dimensional photonic crystal [6989-12]

J. Zaghdoudi, R. Kuszelewicz, M. Kanzari, B. Rezig, Ecole Nationale d'Ingénieurs de Tunis (Tunisia)

\section{SESSION $3 \quad$ PROBING PHOTONIC CRYSTALS}

6989 OG Asymmetry reversal and waveguide modes in photonic crystal slabs [6989-15]

E. F. C. Driessen, P. O. M. Heemskerk, D. Stolwijk, Leiden Univ. (Netherlands);

E. W. J. M. van der Drift, Delft Univ. of Technology (Netherlands); M. J. A. de Dood, Leiden

Univ. (Netherlands)

6989 ol Optimization of a negative index photonic crystal slab at optical wavelength [6989-17]

N. Fabre, X. Mélique, O. Vanbésien, D. Lippens, Institut d'Electronique de Microélectronique et de Nanotechnologie (France)

6989 0J Arrays of selectively grown GaN micro-pyramids: photonic and optical-frequency phonon properties [6989-18]

D. Coquillat, M. Le Vassor d'Yerville, P. Arcade, M. Kazan, Univ. Montpellier II (France);

C. Liu, I. M. Watson, P. R. Edwards, R. W. Martin, Univ. of Strathclyde (United Kingdom);

H. M. H. Chong, R. M. De La Rue, Univ. of Glasgow (United Kingdom)

6989 OK Approaching quantitative optical diffraction analysis of crystal lattices in opal films [6989-19]

W. Khunsin, Tyndall National Institute, Univ. College Cork (Ireland); C. M. Sotomayor Torres, Tyndall National Institute, Univ. College Cork (Ireland), Catalan Institute for Research and Advanced Studies (Spain), and Catalan Institute of Nanotechnology (Spain);

S. G. Romanov, Tyndall National Institute, Univ. College Cork (Ireland) and loffe Physical Technical Institute (Russia)

\section{SESSION 4 LIGHT EMISSION I}

6989 OL Analysis of the emission characteristics of photonic crystal LEDs (Invited Paper) [6989-21] Ch. Wiesmann, Osram Opto Semiconductors GmbH (Germany) and Univ. Regensburg (Germany); K. Bergenek, N. Linder, Osram Opto Semiconductors GmbH (Germany); U. T. Schwarz, Univ. Regensburg (Germany)

6989 OM Control of mode volume and radiation dynamics of a slow-light-mode in a quasi-3D photonic crystal configuration (Invited Paper) [6989-22]

B. Ben Bakir, S. Boutami, Ecole Centrale de Lyon (France) and CEA-LETI Minatec (France);

C. Seassal, X. Letartre, J.-L. Leclercq, P. Viktorovitch, Ecole Centrale de Lyon (France);

M. Zussy, L. Di Cioccio, J.-M. Fedeli, CEA-LETI Minatec (France)

6989 ON Tuning of spontaneous emission in photonic crystals by resonant energy transfer and magnetic fields [6989-23]

W. Libaers, K. Baert, R. A. L. Vallée, B. Kolaric, K. Clays, Katholieke Univ. Leuven (Belgium)

$6989 \mathrm{OP} \quad 1.5 \mu \mathrm{m}$ photoluminescence of $\mathrm{Er}^{3+}$ in opal based photonic crystals [6989-25]

G. Emelchenko, E. Steinman, V. Masalov, A. Tereshchenko, A. Bazhenov, Institute of Solid State Physics (Russia); A. Grishin, Royal Institute of Technology (Sweden) 
$69890 Q \quad$ Photonic crystal laser based on activated glass [6989-26]

O. N. Kozina, Institute of Radio-Engineering and Electronics (Russia); L. A. Melnikov, Saratov State Univ. (Russia)

\section{SESSION $5 \quad$ LIGHT EMISSION II}

6989 OR Non-linear effects in luminescence of ZnO inverted opals (Invited Paper) [6989-27]

W. Khunsin, P. Lovera, G. Redmond, Tyndall National Institute, Univ. College Cork (Ireland); C. M. Sotomayor Torres, Tyndall National Institute, Univ. College Cork (Ireland), Catalan Institute for Research and Advanced Studies (Spain), and Catalan Institute of Nanotechnology (Spain); M. Scharrer, L. Aagesen, R. P. H. Chang, Northwestern Univ. (USA); S. G. Romanov, Tyndall National Institute, Univ. College Cork (Ireland) and Ioffe Physical Technical Institute (Russia)

6989 OS Control of pattern formation in a single feedback system by photonic bandgap structures [6989-28]

N. Marsal, G. Montemezzani, M. Sciamanna, D. Wolfersberger, Univ. Paul Verlaine-Metz and SUPELEC (France); D. N. Neshev, Australian National Univ. (Australia)

6989 OT Thermal emission properties of 2D and 3D silicon photonic crystals [6989-29]

B. Gesemann, S. L. Schweizer, R. B. Wehrspohn, Martin-Luther-Univ. of Halle-Wittenberg (Germany)

6989 OU Impact of dry-etching induced damage in InP-based photonic crystals [6989-30] A. Berrier, Y. Shi, J. Siegert, S. Marcinkevicius, S. He, S. Anand, Royal Institute of Technology (Sweden)

6989 OV Photonic crystal slab mirrors for an ultimate vertical and lateral confinement of light in vertical Fabry Perot cavities [6989-31]

S. Boutami, B. Ben Bakir, Ecole Centrale de Lyon (France) and CEA-DRT/LETI (France);

X. Letartre, J.-L. Leclercq, P. Viktorovitch, Ecole Centrale de Lyon (France)

6989 OW Vertical microcavities based on photonic crystal mirrors for III-V/Si integrated microlasers [6989-32]

L. Ferrier, S. Boutami, F. Mandorlo, X. Letartre, P. Rojo Romeo, P. Viktorovitch, Ecole Centrale de Lyon (France); P. Gilet, B. Ben Bakir, P. Grosse, J.-M. Fedeli, A. Chelnokov, CEA-LETI Minatec (France)

6989 OX A comparative study of directive emission from photonic quasicrystals [6989-33] A. Micco, V. Galdi, V. Pierro, Univ. of Sannio (Italy); A. Della Villa, F. Capolino, Univ. of Siena (Italy); S. Enoch, G. Tayeb, Institut Fresnel, CNRS, Aix-Marseille Univ. (France)

6989 OY Static and dynamic properties of vertical-cavity surface-emitting semiconductor lasers with incorporated two-dimensional photonic crystals [6989-34] P. S. Ivanov, Y. Zhu, M. J. Cryan, P. J. Heard, Y.-L. D. Ho, J. M. Rorison, Univ. of Bristol (United Kingdom) 
698910 Plasmonic excitations in ordered assemblies of metallic nanoshells [6989-36]

N. Stefanou, C. Tserkezis, G. Gantzounis, Univ. of Athens (Greece)

698911 Tunable Fabry-Pérot THz filter with sub-wavelength grating mirrors [6989-37]

T. Goebel, D. Schoenherr, C. Sydlo, Technische Univ. Darmstadt (Germany); A. Roggenbuck,

A. Deninger, Toptica Photonics AG (Germany); P. Meissner, H.-L. Hartnagel, Technische Univ.

Darmstadt (Germany)

698912 Coupled surface states in one- and two-dimensional frequency dependent photonic crystals [6989-38]

M. Bergmair, K. Hingerl, Johannes Kepler Univ. Linz (Austria)

698913 Dynamic optical gratings in two layer system: ferroelectric and nanostructured metal film [6989-39]

N. Kukhtarev, T. Kukhtareva, G. Stargell, Alabama A\&M Univ. (USA)

\section{SESSION 8 NLO/SWITCHING I}

698915 Second harmonic localization in nonlinear photonic crystals (Invited Paper) [6989-41]

E. Centeno, C. Ciraci, D. Felbaca, D. Cassagne, Univ. Montpellier II (France)

698916 Nonlinear photonic structures for all-optical deflection [6989-42]

T. Ellenbogen, A. Ganany-Padowicz, A. Arie, Univ. of Tel-Aviv (Israel)

698917 Quick and non-invasive method for characterisation of profiles of nano-photonics structures [6989-43]

S. Lis, Wroclaw Univ. of Technology (Poland); R. Dylewicz, Univ. of Glasgow (United Kingdom); J. Myśliwiec, A. Miniewicz, S. Patela, Wroclaw Univ. of Technology (Poland)

698919 Few-cycle pulses interactions in nonlinear photonic crystals with managed dispersion [6989-45]

V. E. Lobanov, A. P. Sukhorukov, Lomonosov Moscow State Univ. (Russia)

\section{SESSION 9 FABRICATION/DEVICES I}

6989 1A High-transmission 1D photonic crystal/photonic wire multiple cavity structures based on silicon-on-insulator [6989-46]

A. R. Md Zain, N. P. Johnson, R. M. De La Rue, Univ. of Glasgow (United Kingdom)

6989 1B Quality factor optimization of photonic crystal cavities through multiple multipole expansion technique and power loss integral [6989-47]

R. Jannesary, S. Zamiri, Johannes Kepler Univ. Linz (Austria); A. D. Mazaheri, Univ. of Isfahan (Iran); A. Hamidipour, Sahand Univ. of Technology (Iran); A. Mohtashami, K. Hingerl, Johannes Kepler Univ. Linz (Austria); J. Zarbakhsh, Johannes Kepler Univ. Linz (Austria) and Automobil- und Industrie-Elektronik GmbH (Germany) 
6989 1D Novel design of photonic crystal dense wavelength multiplexer based on nonlinear effect [6989-49]

W. Aroua, F. AbdelMalek, Univ. of Tunis (Tunisia); S. Haxha, Univ. of Kent (United Kingdom); H. Bouchriha, Univ. of Tunis (Tunisia)

6989 IF All-optical diode action with Thue-Morse quasiperiodic photonic crystals [6989-52] F. Biancalana, Cardiff Univ. (United Kingdom)

6989 1G Switching of electromagnetic eigenwaves in metastructures [6989-53]

E. Ya. Glushko, Institute of Semiconductor Physics (Ukraine)

\section{SESSION 11 FABRICATION/DEVICES II}

$698911 \quad$ Efficient coupling of light between single-mode waveguides and supercollimating photonic crystals [6989-55]

K. Vynck, E. Centeno, M. Le Vassor d'Yerville, D. Cassagne, Univ. Montpellier II (France)

$69891 \mathrm{~J} \quad$ Novel multiplexer/demultiplexer based on photonic crystal superprism [6989-56]

W. Aroua, F. AbdelMalek, Univ. of Tunis (Tunisia); S. Haxha, Univ. of Kent (United Kingdom);

H. Bouchriha, Univ. of Tunis (Tunisia)

\section{SESSION 12 FABRICATION/DEVICES III}

698910 Design criteria and 3D FEM modeling of air hole photonic crystal [6989-61]

A. Massaro, V. Errico, R. Cingolani, A. Passaseo, M. De Vittorio, Univ. del Salento (Italy)

6989 1P Temperature tuning of ultra-high Q/V SOI microcavities [6989-62]

P. Velha, CEA-Grenoble (France), Institut d'Optique, CNRS (France), and CEA-LETI-Minatec (France); C. Jocteur, CEA-Grenoble (France) and CEA-LETI-Minatec (France); E. Picard, T. Charvolin, E. Hadji, CEA-Grenoble (France); J. C. Rodier, P. Lalanne, Institut d'Optique, CNRS (France); D. Peyrade, CEA-LETI-Minatec (France)

$69891 Q \quad$ Rod shape optimization in photonic crystal bends by the finite element method [6989-63] B. M. A. Rahman, A. Agrawal, K. T. V. Grattan, City Univ. (United Kingdom); S. S. A. Obayya, Univ. of Leeds (United Kingdom)

6989 1R Photonic crystal waveguide arrays [6989-64]

H. Kurt, TOBB Univ. of Economics and Technology (Turkey)

6989 is Photonic crystal-based WDM filter for integrated optical triplexer transceiver [6989-65] D.-S. Park, J.-H. Kim, B.-H. O, S.-G. Park, E.-H. Lee, S. G. Lee, Inha Univ. (South Korea) 
6989 IT Complete photonic band gap in icosahedral quasicrystals with a body-centered six-dimensional lattice [6989-67]

P. N. Dyachenko, Image Processing Systems Institute (Russia); V. E. Dmitrienko, A.V. Shubnikov Institute of Crystallography (Russia); Yu. V. Miklyaev, South-Ural State Univ. (Russia); V. S. Pavelyev, Image Processing Systems Institute (Russia)

$69891 \mathrm{U}$ Holographic fabrication of hierarchical nanostructures using microprism array toward optofluidic integration [6989-70]

S.-K. Lee, H. S. Park, S. G. Park, Korea Advanced Institute of Science and Technology (South Korea); J. H. Moon, Sogang Univ. (South Korea); S.-M. Yang, Korea Advanced Institute of Science and Technology (South Korea)

6989 IV Dispersion and symmetry properties of anisotropic photonic crystals of arbitrary geometry and dimension [6989-69]

I. A. Khromova, Saratov State Univ. (Russia) and Public Univ. of Navarre (Spain);

L. A. Melnikov, Saratov State Univ. (Russia)

6989 1X Periodic NLC phase grating for tuneable PBG applications [6989-68]

R. Ghannam, American Univ. in Cairo (Egypt); A. Georgiou, Cambridge Univ. (United Kingdom)

Author Index 


\title{
Conference Committee
}

\author{
Symposium Chairs
}

Hugo Thienpont, Vrije Universiteit Brussel (Belgium)

Giancarlo C. Righini, Istituto di Fisica Applicata Nello Carrara, CNR (Italy)

Patrick P. Meyrueis, Université Lovis Pasteur (France)

Conference Chairs

Richard M. De La Rue, University of Glasgow (United Kingdom)

Ceferino López, Consejo Superior de Investigaciones Científicas (Spain)

Michele Midrio, Università degli Studi di Udine (Italy)

Pierre Viktorovitch, Ecole Centrale de Lyon (France)

Program Committee

Lucio C. Andreani, Università degli Studi di Pavia (Italy)

Wim Bogaerts, Universiteit Gent (Belgium)

Roberto Cingolani, Istituto Nazionale per la Fisica della Materia, CNR (Italy)

Claudio Conti, Università degli Studi di Roma La Sapienza (Italy)

Dominique Coquillat, Université Montpellier II (France)

René M. de Ridder, Universiteit Twente (Netherlands)

Andrea Di Falco, Università degli Studi di Roma Tre (Italy)

Stefan Enoch, Institut Fresnel, CNRS (France)

Jean-Michel Gérard, CEA Grenoble (France)

Romuald Houdré, Ecole Polytechnique Fédérale de Lausanne (Switzerland)

Jørn M. Hvam, Danmarks Tekniske Universitet (Denmark)

Florian Kulzer, Institut de Ciències Fotòniques (Spain)

Juan Ariel Levenson, Laboratoire de Photonique et de Nanostructures, CNRS (France)

Stefan Linden, Forschungszentrum Karlsruhe (Germany)

Olivier M. Parriaux, Université Jean Monnet Saint-Etienne (France)

Adriana Passaseo, Università degli Studi di Lecce (Italy)

Pablo A. Postigo, Consejo Superior de Investigaciones Científicas (Spain)

Min Qiu, The Royal Institute of Technology (Sweden)

Sergei G. Romanov, Tyndall National Institute (Ireland)

Alexei Tchelnokov, CEA-LETI (France)

Ulrike Woggon, Universität Dortmund (Germany)

Alejandro M. Yacomotti, Laboratoire de Photonique et de

Nanostructures, CNRS (France) 
Session Chairs

13 3D Photonic Crystals and Volume 2D Photonic Crystals

Ceferino López, Consejo Superior de Investigaciones Científicas (Spain)

Florian Kulzer, Institut de Ciències Fotòniques (Spain)

2 Quasi Photonic Crystals

Michele Midrio, Università degli Studi di Udine (Italy)

Concita Sibilia, Università degli Studi di Roma La Sapienza (Italy)

3 Probing Photonic Crystals

Sergei G. Romanov, Tyndall National Institute (Ireland)

$4 \quad$ Light Emission I

Pierre Viktorovitch, Ecole Centrale de Lyon (France)

Lucio C. Andreani, Università degli Studi di Pavia (Italy)

$5 \quad$ Light Emission II

Romuald Houdré, Ecole Polytechnique Fédérale de Lausanne

(Switzerland)

$6 \quad$ Light Emission III

Romuald Houdré, Ecole Polytechnique Fédérale de Lausanne

(Switzerland)

Juan Ariel Levenson, Laboratoire de Photonique et de Nanostructures, CNRS (France)

$7 \quad$ Metallic Photonic Crystals

Stefan Linden, Forschungszentrum Karlsruhe (Germany)

Philippe Lalanne, Institut d'Optique, CNRS (France)

$8 \quad \mathrm{NLO} /$ Switching I

Alessandro Massaro, Istituto Nazionale per la Fisica della Materia, CNR (Italy)

9 Fabrication/Devices I

Alessandro Massaro, Istituto Nazionale per la Fisica della Materia, CNR (Italy)

10 NLO/Switching II

Alejandro M. Yacomotti, Laboratoire de Photonique et de

Nanostructures, CNRS (France)

Xavier Letartre, Ecole Centrale de Lyon (France)

11 Fabrication/Devices II

Pieter Dumon, Universiteit Gent (Belgium)

Alessandro Massaro, Istituto Nazionale per la Fisica della Materia, CNR (Italy)

12 Fabrication/Devices III

René M. De Ridder, Universiteit Twente (Netherlands) 